\title{
Measurement of photon production cross sections also in association with jets with the ATLAS detector*
}

\author{
Sebastien Prince \\ Department of Physics, McGill University, Montreal, Canada \\ on behalf of the ATLAS Collaboration
}

\begin{abstract}
The production of prompt isolated photons at hadron colliders provides a stringent test of perturbative QCD and can be used to probe the proton structure. The production of prompt photons in association with jets provides an additional testing ground for perturbative QCD with a hard colourless probe less affected by hadronisation effects than jet production. At the LHC, the ATLAS collaboration has performed precise measurements of the inclusive production of isolated prompt photons at a centre-of-mass energy of $13 \mathrm{TeV}$. The dynamics of isolated-photon plus jet production in $p p$ collisions at a centre-of-mass energy of $8 \mathrm{TeV}$ have also been measured. In addition, the integrated and differential cross sections for isolated photon pairs at a centre-of-mass energy of $8 \mathrm{TeV}$ have been measured. The results are compared with state-of-the art theory predictions at next-to-leading order in perturbative QCD.
\end{abstract}

Keywords: ATLAS, Standard Model, QCD, photon, jet, diphoton

\section{Introduction}

Prompt photons are interesting objects to measure at the LHC since they test perturbative QCD (pQCD) in a unique way. Being colourless objects, they are less affected by hadronisation effects than jets are, while still being relatively abundant. In addition, as their main production mechanism is through Compton scattering, i.e. through an interaction with the quarks inside the proton, their measurement can help constrain parton distribution functions (PDFs) of the proton. They are also sensitive to non-perturbative QCD effects in the form of parton-to-photon fragmentation functions.

Experimentally, prompt photons are measured as energy deposits in the electromagnetic calorimeter. The shape of the electromagnetic shower they produce must pass some identification criteria to be considered a photon. The main photon background are jets that contain a

\footnotetext{
${ }^{*}$ Talk given at 20th International Conference in Quantum Chromodynamics (QCD 17), 3-7 July 2017, Montpellier, France
}

photon carrying most of the jet energy. To suppress that background, an isolation cut on the activity surrounding the photon is applied.

Theoretically, prompt photons are defined as any photons not coming from hadron decays, for example photons from $\pi^{0} \rightarrow \gamma \gamma$ are not prompt. Prompt photons can be divided into two categories at leading-order (LO) in $\mathrm{pQCD}$ : direct and fragmentation. Direct photons are produced in the hard scatter of the proton-proton collisions. For LO photon production associated with jets, the main diagrams are Compton scattering, $g q \rightarrow \gamma q$, and quark-antiquark annihilation, $q \bar{q} \rightarrow \gamma g$. The latter contribution is suppressed with respect to the former due to the smaller relative size in the proton of the antiquark PDFs to the gluon PDF. For LO diphoton production, the main diagram is quark-antiquark annihilation, $q \bar{q} \rightarrow \gamma \gamma$. A smaller contribution is the next-tonext-to-leading order (NNLO) in pQCD quark box diagram, $g g \rightarrow \gamma \gamma$, which is enhanced by the large gluon PDF of the proton. For both photon plus jet and diphoton production, prompt photons can also be produced 
as initial- or final-state radiation. Although these are formally next-to-leading order (NLO) diagrams, in the collinear limit of the photon being produced close to the parton, non-perturbative fragmentation functions are necessary to avoid divergences. These functions make the diagrams have an effective order similar to the LO direct production processes. As these functions are less understood than $\mathrm{pQCD}$, isolation cuts around the photon are applied in most pQCD calculations to reduce their contribution. These cuts are motivated further from the experimental side which require them to reduce the jet background.

Fiducial cross sections for the above processes are measured with the ATLAS detector [1] and summarised in this document. The way of measuring the cross sections, $\sigma_{\text {fid }}$, starting from a number of recorded events passing fiducial kinematic cuts and photon identification criteria, $N_{\text {data }}$, is given by the following:

$$
\sigma_{\text {fid }}=\frac{N_{\text {data }}-N_{\text {bkg }}}{\mathcal{L}_{\text {int }} \epsilon_{\text {trigger }} C_{\text {unfold }}} .
$$

The background events, $N_{\mathrm{bkg}}$, need to be subtracted from the selected events. This is done by using datadriven methods, such as to be as model-independent as possible. These methods are analysis-dependent but generally make use of an isolation cut to define background events. In the case of the photon production with jets, the isolation cut is on the measured transverse energy within an angular cone of radius 0.4 around the photon: $E_{\mathrm{T}}^{\text {iso }}<4.8 \mathrm{GeV}+0.0042 \times E_{\mathrm{T}}^{\gamma}$. The isolation cut depends on the photon transverse energy, $E_{\mathrm{T}}^{\gamma}$, to ensure a high efficiency in the high $E_{\mathrm{T}}^{\gamma}$ values observed in that mode of production. In the case of diphoton production, a cut on the transverse energy, also in a cone of radius 0.4 , independent of $E_{\mathrm{T}}^{\gamma}$ is applied: $E_{\mathrm{T}}^{\text {iso }}<6 \mathrm{GeV}$. Since the diphoton production vertex can be measured precisely, this is supplemented by an isolation cut on the momentum of tracks associated to that vertex, in a cone of radius $0.2: p_{\mathrm{T}}^{\text {iso }}<2.6 \mathrm{GeV}$.

Due to the limited recording bandwidth compared to the collision rate, only events chosen by the trigger system are recorded. As such, the number of selected events needs to be corrected by the trigger efficiency. For the measurements presented in this document, the fiducial selection was designed to only make use of the trigger system in the phase-space region where it is fully efficient. Thus in these cases, the $\epsilon_{\text {trigger }}$ parameter can essentially be ignored. The events are then unfolded, with $C_{\text {unfold }}$, for any detector inefficiency or resolution effects such as to be comparable to other measurements or theoretical predictions. The translation of number of signal events to cross section value is then done through the measured integrated luminosity of the event dataset, $\mathcal{L}_{\text {int }}$. For differential cross sections binned as function of an observable, an extra step is done relative to equation 1: dividing by the bin width of the observable.

\section{Inclusive photon production at $13 \mathbf{T e V}$}

The inclusive photon cross section includes all processes producing at least one prompt photon. The dominant process is Compton scattering, in which the quark, or jet, is not measured explicitly. Since the measurement is inclusive with respect to the quark type, they are sensitive to the gluon PDF and can help constrain it.

The inclusive photon production cross section in proton-proton collisions at a centre-of-mass energy of $13 \mathrm{TeV}$ and with an integrated luminosity of $3.2 \mathrm{fb}^{-1}$ has been measured with ATLAS [2]. The measurements are differential in $E_{\mathrm{T}}^{\gamma}$ and separated in regions of the absolute value of the photon pseudorapidity, $\left|\eta^{\gamma}\right|$. No measurement is done in the region $1.37<\left|\eta^{\gamma}\right|<1.56$ as it covers the gap between the barrel and endcap of the electromagnetic calorimeter, for which the photon identification efficiency is not as well-measured as in the rest of the calorimeter. The measurements are presented in Figure 1. They are compared to the JETPHOx calculations, using the MMHT2014 PDF set. JETPHOx has NLO accuracy and includes the contributions of both direct and fragmentation photons. The total uncertainty on the theory predictions is about $10-15 \%$, which is dominated by the uncertainty related to the choice of the values for the renormalisation, factorization and fragmentation scales. The experimental uncertainty is smaller in comparison at $5-10 \%$.

The agreement between the predictions and the measurements is presented in Figure 2 as ratios of the theory to the data. In addition to the predictions using the MMHT2014 PDF set, the CT14 and NNPDF3.0 PDF sets are also considered. They predict cross section values that are similar, in consideration of the total theoretical uncertainty. Overall, there is an adequate agreement between the predictions and the data as, although the former tends to consistently underestimate the latter, the theoretical uncertainties cover the difference between the two. NNLO predictions, with reduced scale uncertainties compared to NLO predictions, would provide a more stringent test of $\mathrm{pQCD}$.

NNLO predictions for direct photons have been made public [3] at a similar time as the publication of these measurements. The NNLO predictions are compared to the $8 \mathrm{TeV}$ inclusive photon cross section measurements [4], for which the NLO predictions showed a similar agreement and underestimation as for the results at 


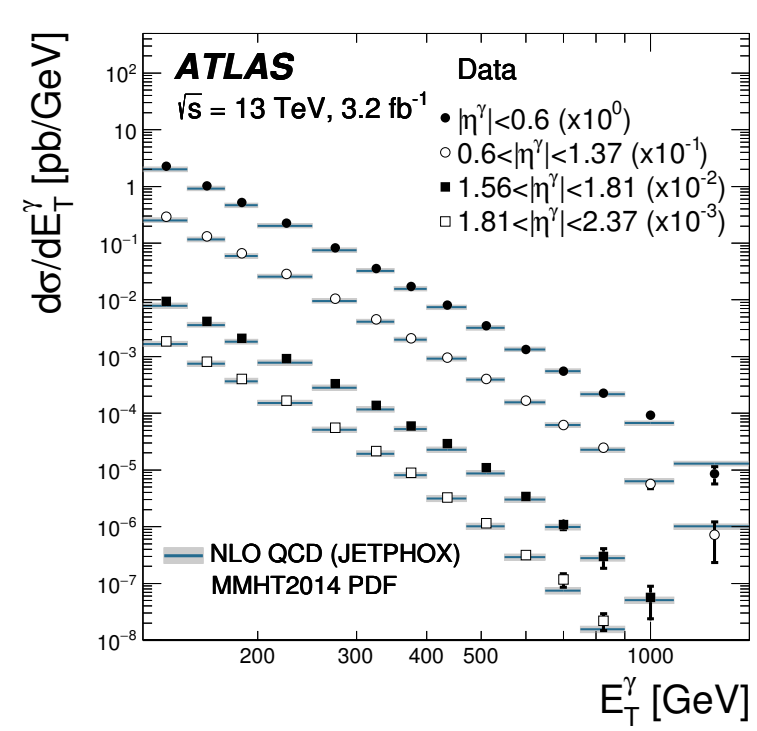

Figure 1: Differential cross section of the inclusive photon production as function of $E_{\mathrm{T}}^{\gamma}$. The measurements are separated in four regions of the absolute value of the photon pseudorapidity. The NLO theory predictions from JETPHOX are compared to the data [2].

$13 \mathrm{TeV}$. The NNLO predictions indeed reduce the theoretical uncertainties such that the total theoretical uncertainty is of the same size as the measurement uncertainty. They also predict larger values for the cross sections such that the theory and data are still in agreement, despite the smaller theoretical uncertainties.

\section{Photon production associated with jet(s) at $8 \mathrm{TeV}$}

The dominant processes for the production of photon associated with jets are similar to that of the inclusive photon processes, except that the jets are measured explicitly. Measuring the jets allows for new observables as function of which the cross section can be measured differentially. These observables allow to test pQCD in new ways. The production of photons with at least one, at least two and at least three jets has been measured with ATLAS in proton collisions at $8 \mathrm{TeV}$, using a dataset with $20.2 \mathrm{fb}^{-1}$ of integrated luminosity [5]. The jets are reconstructed using the anti- $k_{t}$ algorithm with a parameter of $R=0.6$.

The measurements involving the production of a photon with at least one jet are performed differentially as function of several observables: $E_{\mathrm{T}}^{\gamma}, p_{\mathrm{T}}^{\text {jet1 }}, m^{\gamma \text {-jet1 }}$ and $\left|\cos \theta^{*}\right|$, respectively the photon transverse energy, the transverse momentum of the leading jet, defined as the jet having the largest transverse momentum value of

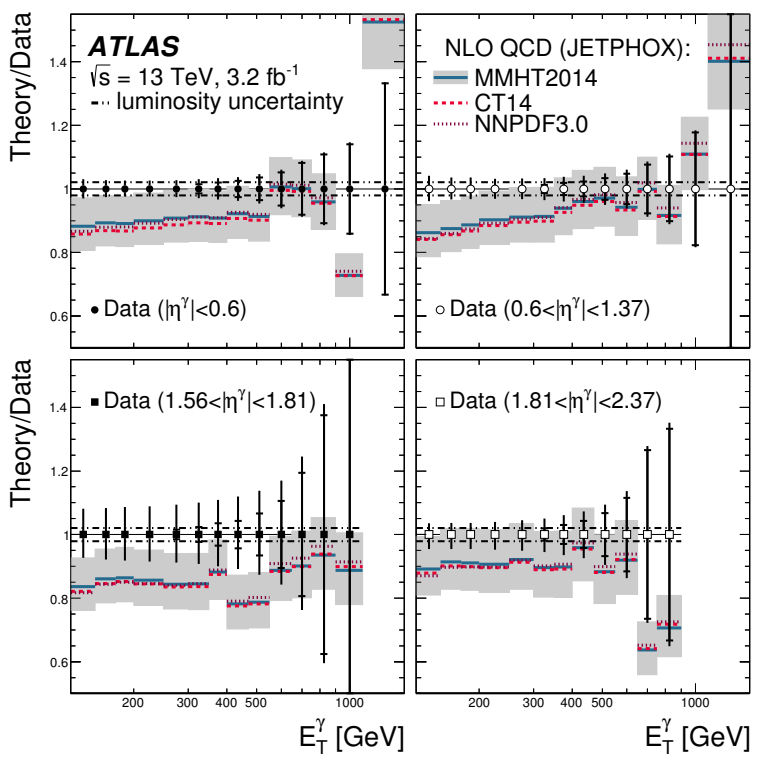

Figure 2: Ratio of theory predictions to measurements of the inclusive photon cross section, differential in $E_{\mathrm{T}}^{\gamma}$ and separated in four regions of the absolute value of the photon pseudorapidity. The theory predictions come from the NLO calculations of JеTPHOx [2].

the event, the invariant mass of the photon and leading jet and the absolute value of the cosine of the scattering angle in the centre-of-mass frame, equivalently $\cos \theta^{*}=\tanh \left[\left(\eta^{\gamma}-y^{\text {jet}}\right) / 2\right]$. Figure 3 shows the differential cross section measurement as function of the invariant mass of the photon-jet system. Cuts on $\left|\cos \theta^{*}\right|$ and $\left|\eta^{\gamma}+y^{\text {jet1 }}\right|$ are applied to ensure the measurement is not biased by the fiducial cuts on the event kinematics. Similarly to the results of the inclusive photon cross section, the measurement is compared to the JетPHOx NLO calculations. Again, the total uncertainty on the predictions of about $10-15 \%$, dominated by the scale uncertainty, is larger than that on the measurement, which is about $5 \%$ in most of the measured $E_{\mathrm{T}}^{\gamma}$ range, i.e. below 1.5 TeV. The data and predictions agree within uncertainties. Although these observations are specific to the measurement as function of the invariant mass, they are representative of the results as function of the other observables.

The $\left|\cos \theta^{*}\right|$ observable is interesting as the contribution to the cross section from the $t$-channel diagrams is expected to scale differently depending on the spin of the exchanged particle. For direct processes, in which a spin- $1 / 2$ particle is exchanged, a quark, the scaling is expected to follow $1 /\left(1-\left|\cos \theta^{*}\right|\right)$ as $\left|\cos \theta^{*}\right| \rightarrow 1$, whereas for fragmentation processes, in which a spin-1 particle is exchanged, a gluon, the scaling is expected to 


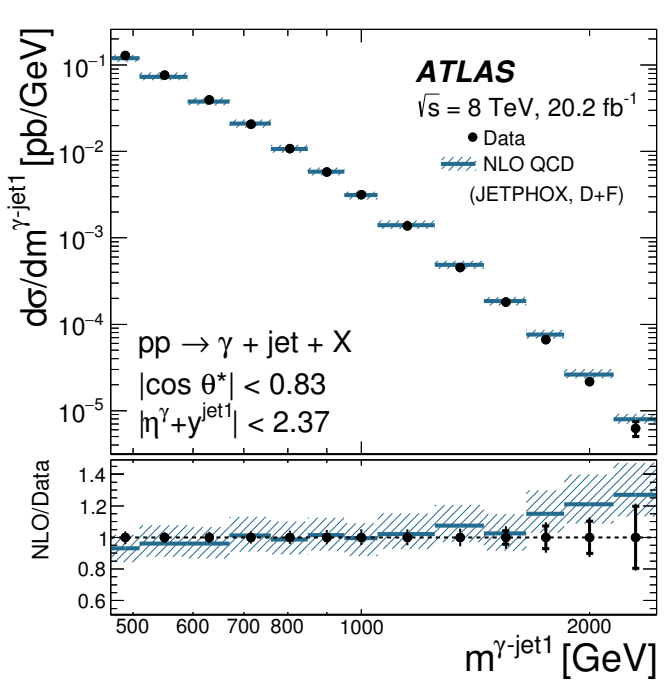

Figure 3: Differential cross section of the photon production associated with at least one jet as function of the invariant mass of the photon-jet system. The ratio of the NLO theory prediction from JETPHox to the measurement is also displayed underneath [5].

follow $1 /\left(1-\left|\cos \theta^{*}\right|\right)^{2}$ as $\left|\cos \theta^{*}\right| \rightarrow 1$. Figure 4 shows the differential cross sections as function of $\left|\cos \theta^{*}\right|$, in several regions of the value of the invariant mass. The LO predictions using JETPHOx for the separate direct and fragmentation contributions are compared to the measurements. This is done at LO as at NLO the two contributions are indistinguishable. In all regions, it is observed that the shape of the measurement follows more closely the direct photon contribution. This observation follows the expectation that the direct photon processes contribute more than the fragmentation processes.

Additional observables become available when measuring the cross section of the photon production in association with at least two jets. The ATLAS measurement considered the following observables: $E_{\mathrm{T}}^{\gamma}, p_{\mathrm{T}}^{\text {jet2 }}$, $\Delta \phi^{\gamma \text {-jet2 }}$ and $\Delta \phi^{\text {jet1-jet2 }}$, respectively the photon transverse energy, the transverse momentum of the subleading jet, the azimuthal angle between the photon and the subleading jet and the azimuthal angle between the leading and subleading jets. The measurements are compared to NLO predictions from BLACKHAT, which in contrast to those from JETPHOx do not include the production of fragmentation photons. Overall, these predictions have a larger uncertainty, which is around $10 \%$ and dominated by the scale uncertainty, than the measurement, about $5 \%$. They provide a good description of the data. Although not displayed here, the only exception is for two $E_{\mathrm{T}}^{\gamma}$ bins at $E_{\mathrm{T}}^{\gamma}>750 \mathrm{GeV}$ for which the predictions overestimate the data beyond the uncertainties.

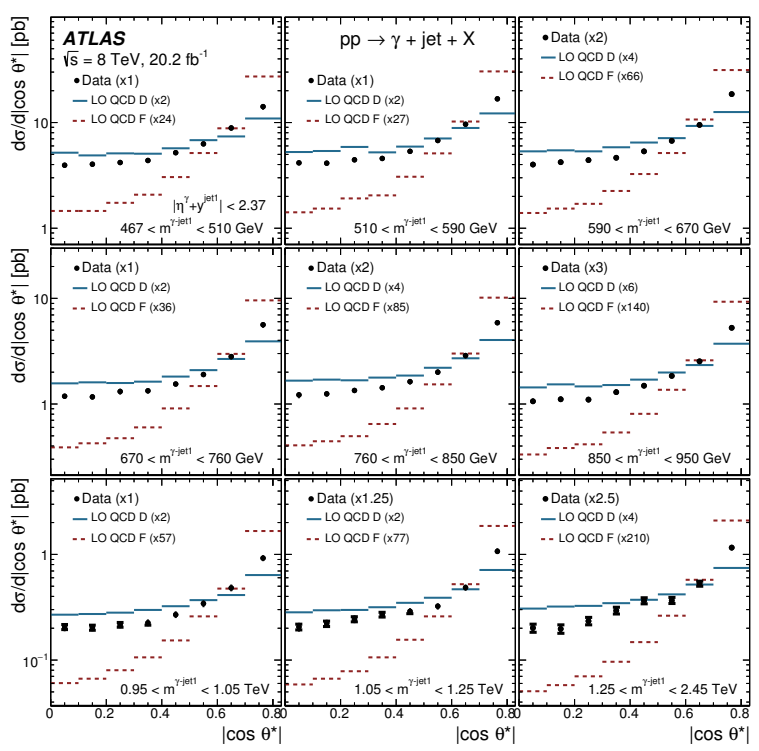

Figure 4: Differential cross section of the photon production associated with at least one jet as function of the absolute value of the cosine of the scattering angle in the centre-of-mass frame. The cross sections are separated in regions of the value of the invariant mass of the photon-jet system. The individual direct and fragmentation components of the photon production as predicted by ЈЕTPHOX at LO are compared to the measurements. A scaling factor is used to multiply the predicted values such that the predicted integrated cross section is equal to the value of the measured integrated cross section, independently in each invariant mass region [5].

In addition to these observables, two further angular quantities were considered: $\beta^{\gamma}$ and $\beta^{\text {jet1 }}$. These angles measure the position of the subleading jet respectively around the prompt photon and the leading jet, when it is close to either of these reference objects. The zero of these angles is defined to be parallel to the beam line, in the direction pointing towards the edge of the detector that is closest to the reference object. The ratio of the differential cross section as function of $\beta^{\text {jet1 }}$ over that as function of $\beta^{\gamma}$ is presented in Figure 5. The measurement is compared to the SHERPA and PyTHIA LO predictions and, although no theoretical uncertainties are provided for these predictions, a good agreement can be seen. The ratio is observed to vary with $\beta$, showing a deficit in the transverse plane of the detector, at $\pi / 2$. To further test this observation, the hypothesis of a constant value for the ratio has been tested and results in a $p$-value of $1.3 \%$. Thus, this variation in the ratio is the first observation that the jet production around the photon is different to that around the leading jet.

The ATLAS measurement of the cross section of the photon production with at least three jets has been done with respect to the following variables: $E_{\mathrm{T}}^{\gamma}, p_{\mathrm{T}}^{\text {jet3 }}$, 


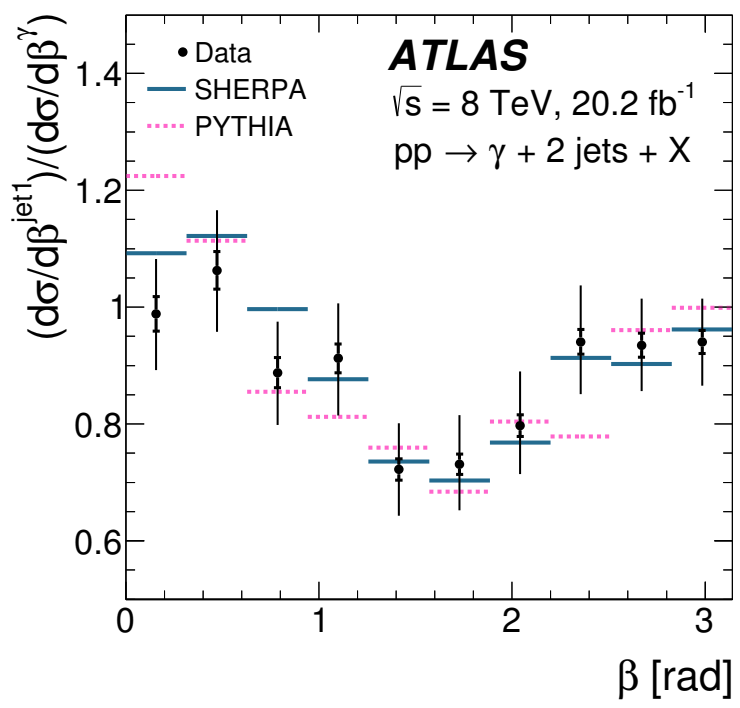

Figure 5: Ratio of the differential cross sections of jet production around the leading jet to that around the prompt photon. The measurement is compared to the LO predictions of SHERPA and PYTHIA [5].

$\Delta \phi^{\gamma \text {-jet3 }}, \Delta \phi^{\text {jet1-jet3 }}$ and $\Delta \phi^{\text {jet2-jet3 }}$, respectively the photon transverse energy, the transverse momentum of the subsubleading jet, the azimuthal angle between the photon and the subsubleading jet, the azimuthal angle between the leading and subsubleading jets and the azimuthal angle between the subleading and subsubleading jets. Similarly to the production of a photon accompanied by at least two jets, the measurements are compared to NLO predictions from BLACKHAT. The predictions have a total uncertainty of a comparable size to that of the measurements, about $10 \%$. They provide a good description of the data.

\section{Diphoton production at $8 \mathrm{TeV}$}

The diphoton production is dominated by the LO quark-antiquark annihilation diagram. Compared to the inclusive photon production, the diphoton production is smaller, because of the suppression of the quarkantiquark diagrams due to the PDFs of the proton and because of the smaller size of the electromagnetic coupling constant compared to the strong coupling constant. Another difference is that the diphoton diagrams have no jets in the final state at LO. For this reason, the diphoton production provides an opportunity to test the production of soft gluons precisely, for which QCD resummation effects are important.

Measurements of diphoton production were performed with ATLAS at $8 \mathrm{TeV}$, using an integrated lu- minosity of $20.2 \mathrm{fb}^{-1}$ [6]. The integrated fiducial cross section is shown in Figure 6. Several theory predictions are compared to the measurement: predictions from Diphox, Resbos, $2 \gamma$ NNLO and Sherpa. Diphox is similar to JетPнох: it calculates at NLO both the direct and fragmentation photon contributions. It also takes into account the contribution from the NNLO quark box diagram. Resbos provides a calculation at NLO of the direct photon contribution and includes the resummation of soft gluons at next-to-next-to-leading-log accuracy. In addition, it includes the quark box diagram and also its higher-order correction in pQCD. $2 \gamma$ NNLO provides a prediction at NNLO of the direct photon contribution. SHERPA, while formally providing a prediction at NLO, includes diphoton diagrams with up to three additional partons in the final-state. The SHERPA predictions are thus sensitive to effects appearing formally at a higher order than NNLO. It also interfaces the calculations to a parton shower, thus giving a better description of the soft gluon emission than a fixed-order calculation. The theory uncertainties on these predictions are dominated by the scale uncertainty and are larger than the experimental uncertainty. They are respectively about $15 \%$, $10 \%$ and $20 \%$ for Diphox, $2 \gamma$ NNLO and SHERPa while the measurement uncertainty is about $5 \%$. No uncertainty was provided by the authors of ResBos. The prediction from SHERPA agrees with the data, but the other predictions tend to underestimate the data.

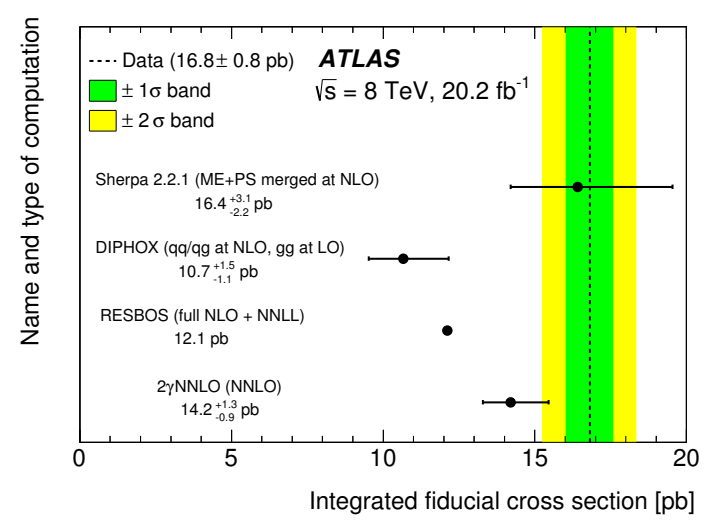

Figure 6: Integrated cross section of the diphoton production. The measurement is compared to the Sherpa, Diphox and Resbos predictions at NLO and to the $2 \gamma$ NNLO prediction at NNLO. No uncertainty is displayed for the ResBos prediction [6].

The diphoton differential cross sections have been measured as function of the following observables, either sensitive to the production of low $p_{\mathrm{T}}$ gluons, such as $p_{\mathrm{T}}^{\gamma \gamma}, \Delta \phi^{\gamma \gamma}, a_{\mathrm{T}}$ and $\phi_{\eta}^{*}$, or to possible new physics effects: $m^{\gamma \gamma}$ and $\left|\cos \theta^{*}\right|$. Other than $a_{\mathrm{T}}$ and $\phi_{\eta}^{*}$, the de- 
scription of these variables in order is the transverse momentum of the diphoton system, the azimuthal angle between the two photons, the invariant mass of the diphoton system and the absolute value of the cosine of the scattering angle in the centre-of-mass frame. The $a_{\mathrm{T}}$ variable is defined as the transverse component of $p_{\mathrm{T}}^{\gamma \gamma}$ with respect to the thrust axis, which is defined as the direction of the vector difference between the transverse momentum of the two photons. When no gluon is emitted, $a_{\mathrm{T}}=0$. Values close to zero thus probe the effect of soft gluon emission. The $\phi_{\eta}^{*}$ variable is defined such as to have the best experimental resolution of the variables sensitive to the production of low $p_{\mathrm{T}}$ gluons, formally in terms of angular variables only. When the two photons have a similar transverse momentum value, the following approximation can be made: $\phi_{\eta}^{*} \approx a_{\mathrm{T}} / m^{\gamma \gamma}$, showing that the region sensitive to soft gluon emission is also close to zero. These differential cross sections are all compared to the same four theory predictions as for the integrated cross section. Overall, their uncertainties are typically the same size as those for the integrated cross section, in most bins.

Figure 7 shows the measured and predicted differential cross sections, and their ratios, as function of $\phi_{\eta}^{*}$. The low $\phi_{\eta}^{*}$ region is well-modelled by ResBos and SHERPa, which both include the effects of soft gluon emission beyond fixed-order in their calculations. DipHox and $2 \gamma$ NNLO however do not model well that region and even predict unphysical negative values in some cases. For larger values of $\phi_{\eta}^{*}$, DipHox and ResBos both underestimate the data, by up to a factor two. $2 \gamma$ NNLO also underestimates the data but the discrepancy is smaller. SHERPA describes well the data for all values of $\phi_{\eta}^{*}$.

The agreement, or lack thereof, seen between the theory predictions and the measurement as function of $\phi_{\eta}^{*}$ is representative of what is seen for the other observables. This points towards a need of higher-order calculations. The low $\phi_{\eta}^{*}$ region shows the importance of including the effects of the soft-gluon resummation in pQCD calculations. The measurement of this phase-space region could serve as a preliminary test for resummation techniques aiming to model more complicated processes, such as colored final state particles.

\section{Conclusion}

Although, photons are not colored and thus non interacting in QCD, their measurement are still useful to probe that theory. They allow to test $\mathrm{pQCD}$ in unique ways at the LHC, since, while they mostly interact with colored particles, they do not hadronise. The inclusive
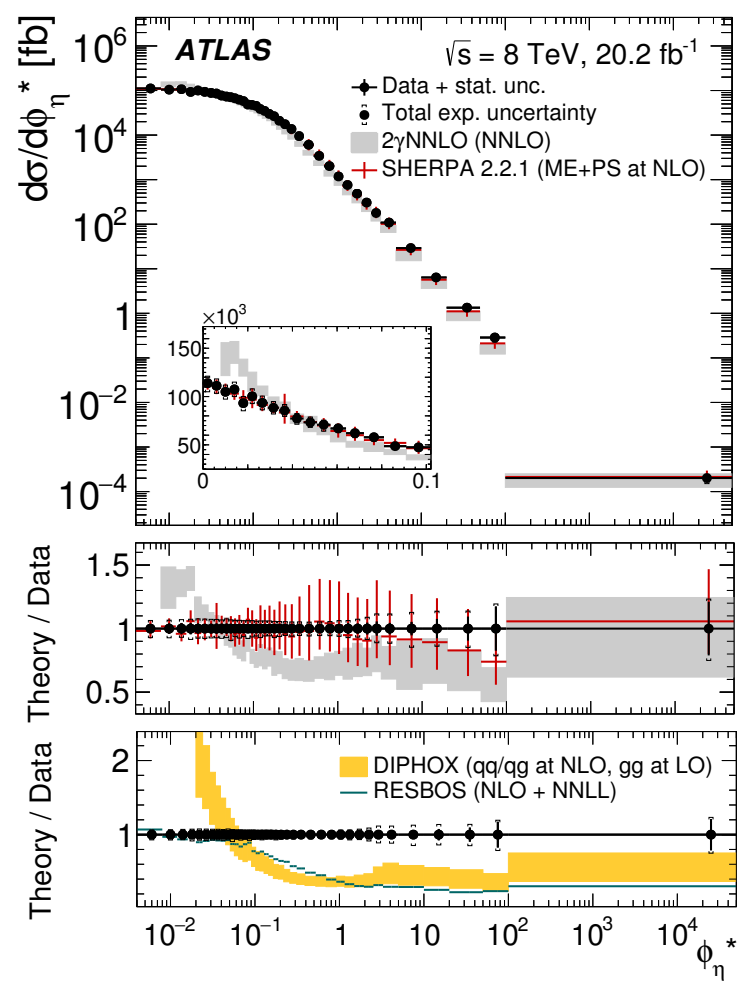

Figure 7: Differential diphoton cross section as function of $\phi_{\eta}^{*}$. Ratios of the NLO theory predictions, from Diphox, REsbos and SHERPA, and NNLO prediction, from $2 \gamma$ NNLO, to the measurements are shown underneath. No uncertainty is displayed for the ResBos predictions [6].

photon production at $13 \mathrm{TeV}$, the photon production associated with at least one, two or three jets at $8 \mathrm{TeV}$ and the diphoton production at $8 \mathrm{TeV}$ are measured with the ATLAS detector. Overall, the NLO theory predictions agree well with the measurements. The theoretical uncertainty, which is dominated by the scale uncertainty, is usually larger than the experimental uncertainty. However, discrepancies can be found in some cases. In general, higher-order pQCD calculations would be necessary for a more stringent test of pQCD.

\section{References}

[1] ATLAS Collaboration, JINST 3 (2008) S08003.

[2] ATLAS Collaboration, Phys. Lett. B 770 (2017) 473, arXiv: 1701.06882 [hep-ex].

[3] J. M. Campbell, R. K. Ellis and C. Williams, Phys. Rev. Lett. 118 (2017) no.22, 222001 arXiv:1612.04333 [hep-ph].

[4] ATLAS Collaboration, JHEP 1608 (2016) 005, arXiv: 1605.03495 [hep-ex].

[5] ATLAS Collaboration, Nucl. Phys. B 918 (2017) 257, arXiv:1611.06586 [hep-ex].

[6] ATLAS Collaboration, Phys. Rev. D 95 (2017) no.11, 112005, arXiv: 1704.03839 [hep-ex]. 